\title{
System description of the defect creation and elimination in the buildings
}

\author{
Dalibor Vytlačili, ${ }^{1, *}$ \\ ${ }^{1}$ Czech Technical University in Prague, Faculty of Civil Engineering, Department of Engineering \\ Informatics, Thákurova 7, 16629 Prague, Czech Republic
}

\begin{abstract}
The paper deals with the dynamics of the system of defects creation in the buildings and also with the possibilities of the defects elimination. The problem is described by the dynamic model that includes key elements influencing the system behaviour. The model is based on the system dynamics method that uses the stock elements and the flow elements which change the stock element values. In the described problem the main stock is the number of the equipment defects. This parameter can be increased by the flow element that depends on the time of using and the load. For the defect elimination are two possibilities: the planned maintenance and the repair of broken parts. Other elements are number of workers available for the elimination of defects and the running cost depending on the maintenance effort. The case of the main system parameters calculation is presented. The model can be used for understanding the importance of the maintenance in the field of facility management.
\end{abstract}

\section{Introduction}

The houses as well as all structures became in past years high complex and it has big influence on the desired quality of the design and construction works in sites. Higher complexity it is possible to see in HVAC systems and other active elements but a similar development is also in the building structure. These factors feature an operation phase. In this phase of building use we perform operations that ensure the quality of the internal environment and all processes related to the building user. Facility management is nowadays common management method which is used for planning and conducting these activities. It makes use of planning tools that are focused on the detail, e.g. scheduling systems and databases, see [1]. Important part of the processes is related to the reduction of the defects.

The correct description of the real behaviour of the system requires the holistic approach. The system approach for describing the system of interdependent elements is necessary. The main elements are the maintenance and the repairs of broken parts. It is dynamic problem that needs dynamic model for the description of the time changes of the variables.

\footnotetext{
*Corresponding author: vytlacil@,fsv.cvut.cz
} 


\section{Method}

The method for understanding the influence of all relevant variables that affect the system is system dynamics. This method uses the stock elements for the description of the variable changes during the time period and the flow elements for the description of rates that change the stock values, [2-3].

The stock value changes are described by Equation 1 .

$$
\operatorname{Stock}(t)=\int_{t_{0}}^{t}[\operatorname{Iflow}(s)-O f l o w(s)] d s+\operatorname{Stock}\left(t_{0}\right)
$$

The design of the cause diagram that presents how entities in the problem are influenced is the first step for creating the system dynamics model. The arrows include also polarity explaining the direction of the change for the next element, see Figure 1.

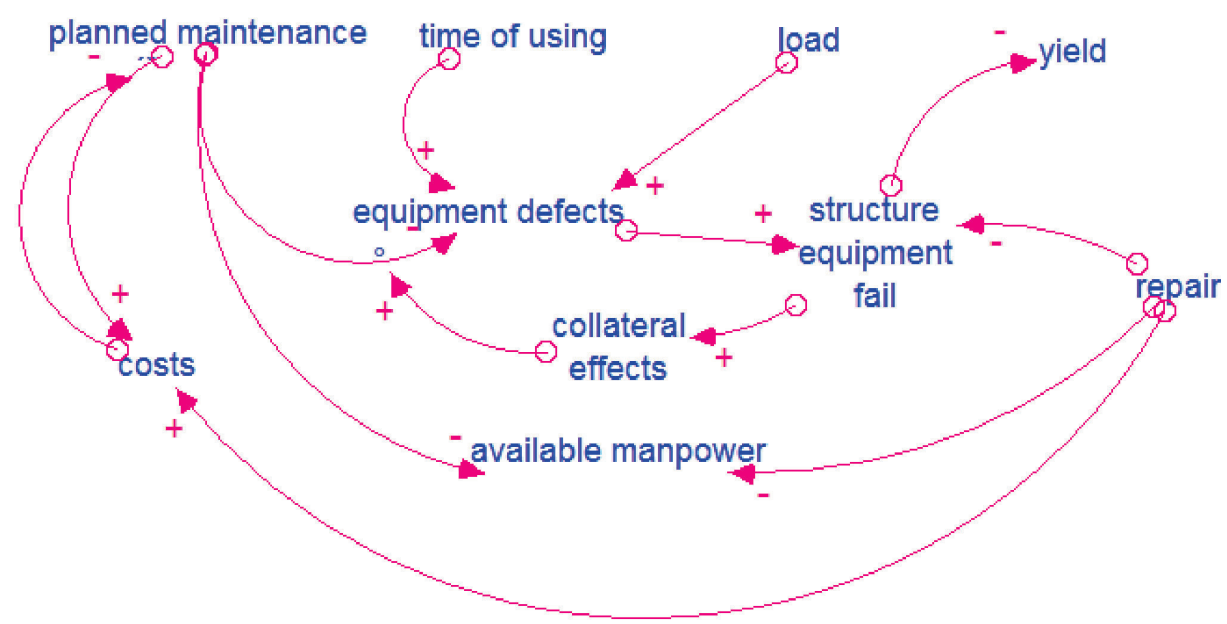

Fig. 1. Cause diagram for described problem.

\subsection{Dynamic model}

The key elements of the system dynamics model concerning the investigated problem are depicted in Figure 2. The main stock is Defect which is influenced by defect creation and two possibilities of the defect elimination: planned maintenance and the repair in case of the broken component, [2]. Presented basic model is the core in the broader model. The model for the real situation has to include subsystems dealing with financing, resources planning and supply line of spare parts for the repairs and the maintenance activities.

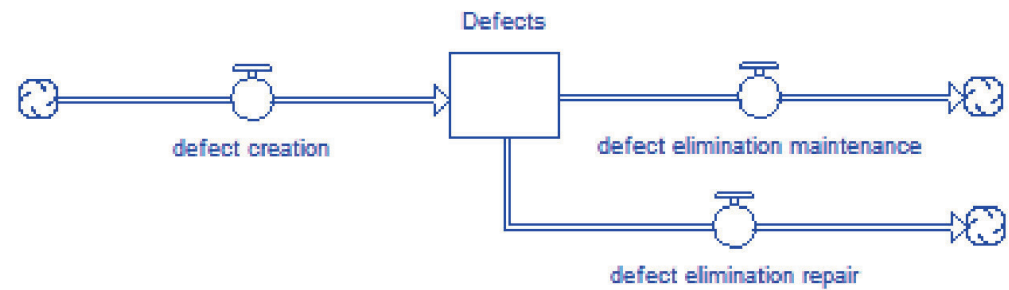

Fig. 2. Basic model of defects creation and elimination. 
The model including described subsystems is presented in Figure 3. The considerable elements are used for manpower dynamics. Active workers maintenance and Active worker repair are numbers of workers used in certain time for planned maintenance or solving ad hoc problems (repairs). Available workers are at this time free for the next work in both kinds of defect elimination. The stock can be negative to demonstrate the situation when the system suffers from manpower shortage.

The elimination is based on the number of workers needed for one defect elimination by means of maintenance and repair (WMD and WRD) and on the time needed for the finishing the task. The defect creation depends on the time of using the component (in some cases the whole building) and on the load (intensity of the building use).

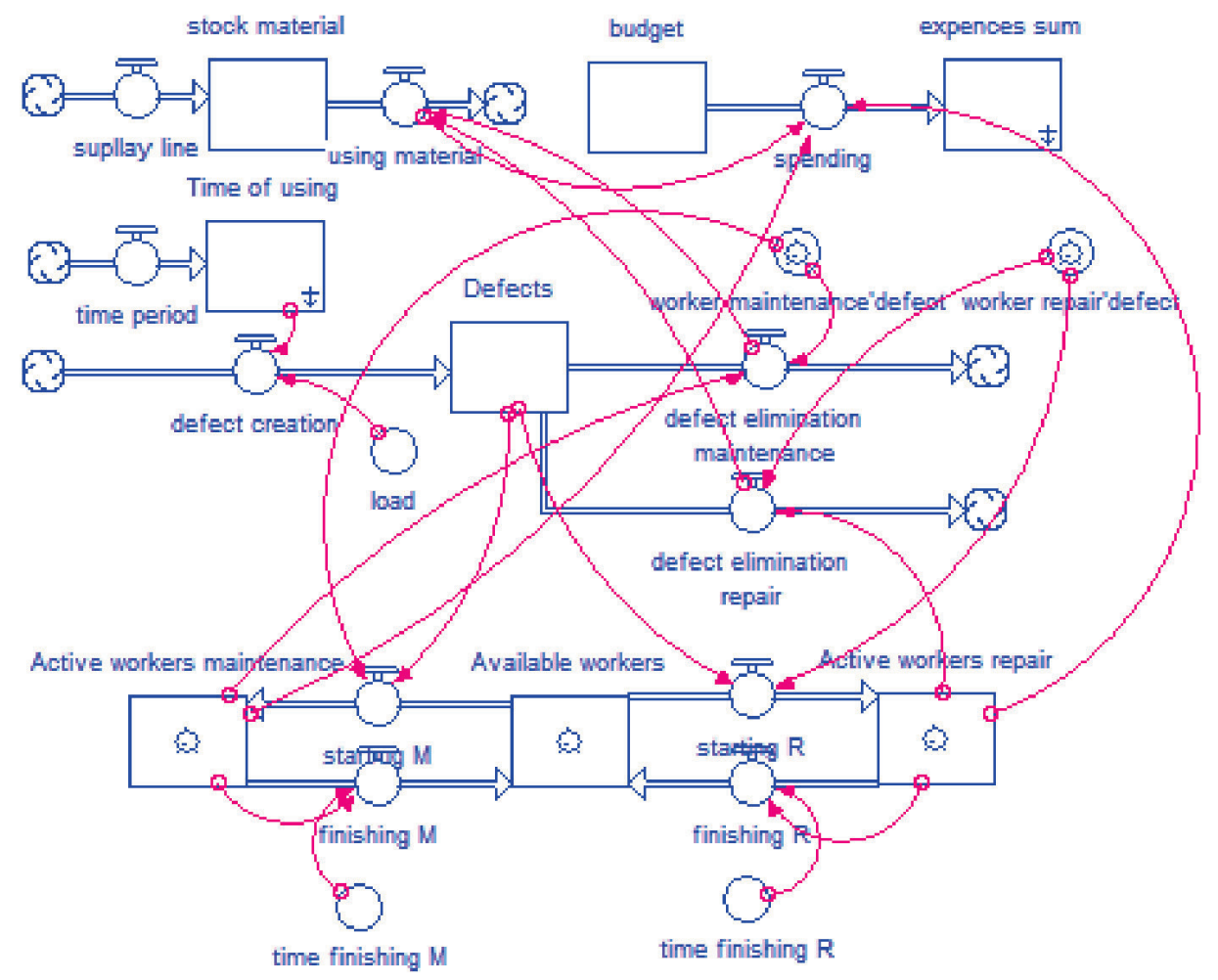

Fig. 3. System dynamics model.

\subsection{Input data}

The simulation is performed for the case of the office building with the floor space $11000 \mathrm{~m}^{2}$. The initial number of workers for ensuring maintenance and repair activities is 4 . The time period is 12 months. The goal of the simulation is to find out the relationship between defects and number of workers in the dynamic environment.

The parameter value Defect is the initial value for the simulation. The initial values for all parameters influence also the dynamics of the system particularly in the case of short time period. The parameter values are in Table 1. 
Table 1. Input values.

\begin{tabular}{|c|c|}
\hline Parameter & Value \\
\hline Active worker maintenance & 2 \\
\hline Active worker repair & 2 \\
\hline Available workers & 0 \\
\hline Defects & 3 \\
\hline WMD & 0.2 \\
\hline WRD & 0.6 \\
\hline
\end{tabular}

\section{Outputs from the simulation}

The output parameter are in Figure 4 and Figure 5. The first group of parameters is focused on the number of defects and flow parameters influencing the stock Defects. In the beginning the high efforts in the defect elimination is evident. In the end of the time period has the influence the defect creation because of the time factor.

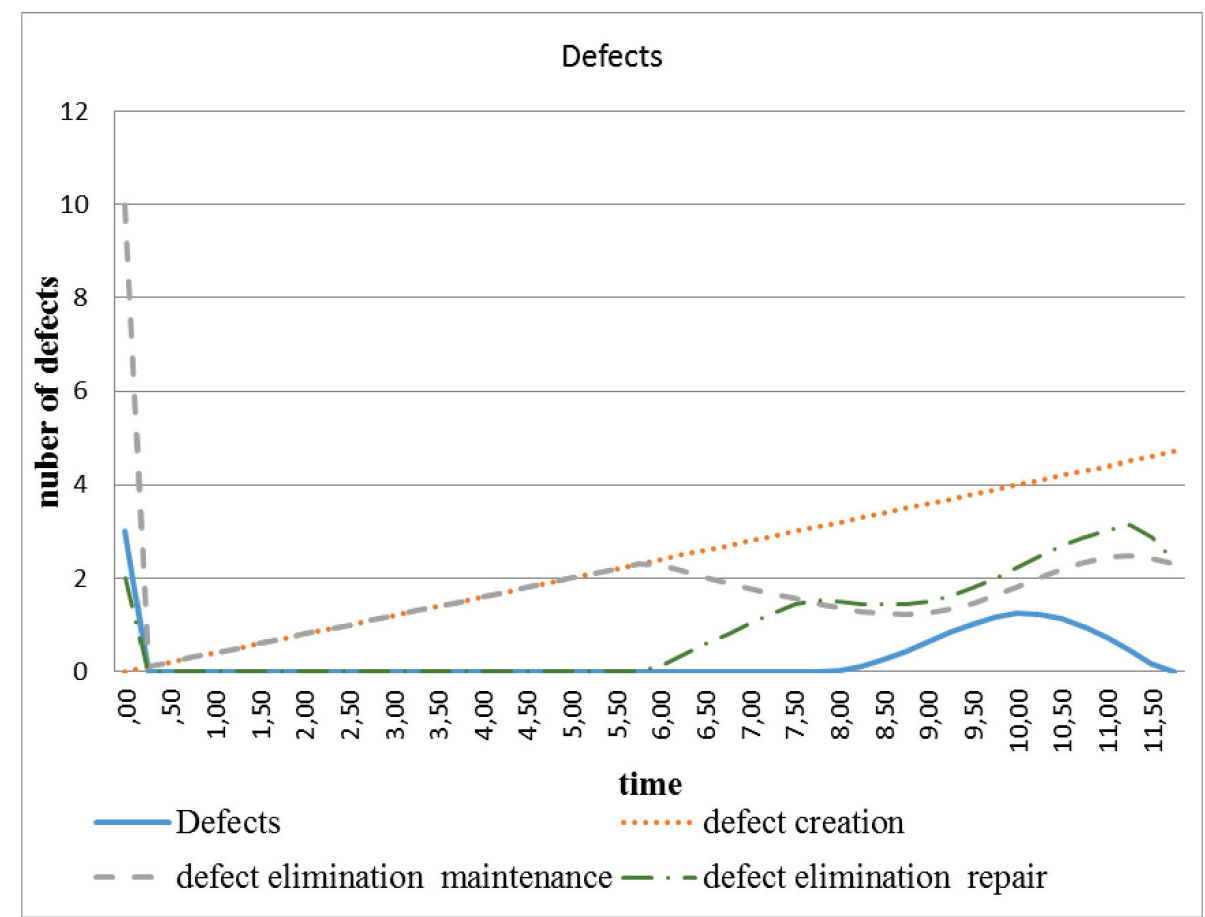

Fig. 4. Defect dynamics.

The manpower dynamics is presented in Figure 5. In the beginning the result shows the worker shortage and the need for more than 4 workers. It means in this time the company providing facility management has to add 1 worker or to use the capacity from the external environment. 


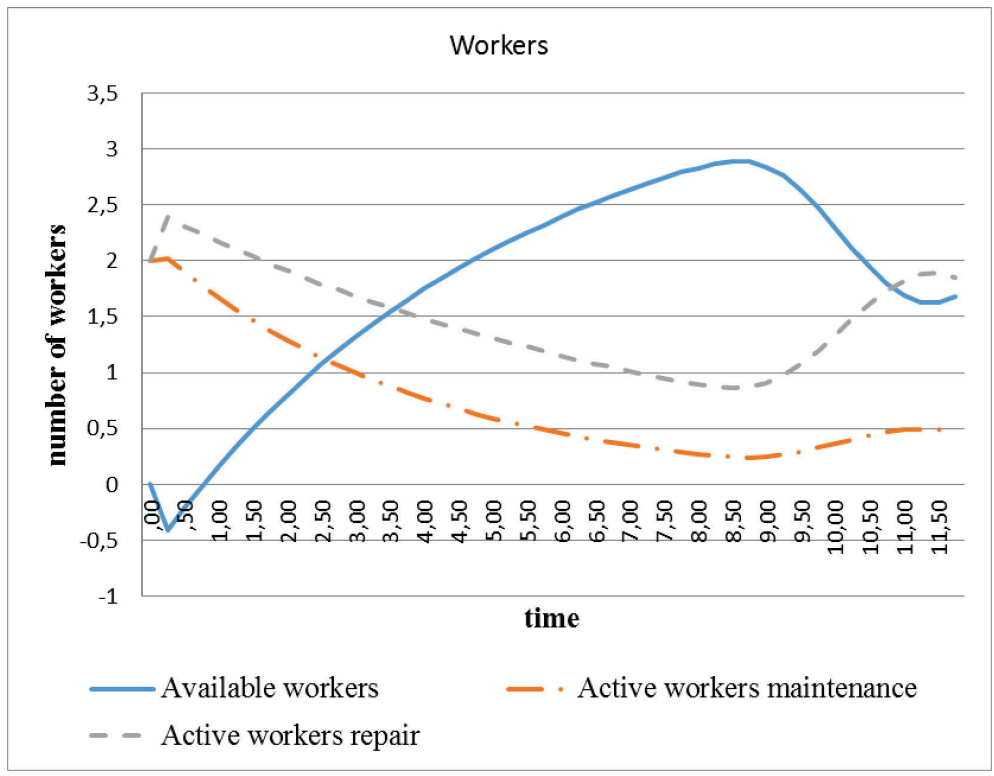

Fig. 5. Manpower dynamics.

\section{Conclusions}

The paper presents the system approach for the calculation of the defects and the description of the dynamics of considered parameters. The model is focused on the dynamics of manpower and does not use the restriction from component supply system or budget.

From the model it is possible to find out:

- Dynamics of the system with interconnected elements;

- Number of workers for maintenance and repair works;

- Dynamics of manpower in the case of the time dependent defect creation;

- Demand for outsourcing.

The next development will be devoted to the design of other subsystems as the stock management for spare parts and the influence of the operating cost on the financial results of the company. It is necessary also consider the changes in revenues in case of repairs that will influence the business outputs of customers (tenants in the building). The broad goal of model using is to find adequate policy for resources planning and company performance improving.

This research has been supported by SGS grant SGS17/122/OHK1/2T/11 Dynamic models of changes in building industry investments.

\section{References}

1. B. Chanter, P. Swallow, Building Maintenance Management (Blackwell Publishing, Oxford, UK, 2007)

2. J.D. Sterman, Business Dynamics: Systems Thinking and Modeling for a Complex World (Irwin/McGraw-Hill, Boston, MA 2000)

3. J. Morecroft, Strategic Modelling and Business Dynamics (Wiley, Chichester, UK, 2008) 
